\title{
Application of Dynamic Regulation to Increase L-Phenylalanine Production in Escherichia colis
}

\author{
Jie $\mathrm{Wu}^{1,2,3 \dagger}$, Yongfei Liu ${ }^{2,3+}$, Sheng Zhao ${ }^{4}$, Jibin Sun ${ }^{2,3}$, Zhaoxia Jin ${ }^{1 *}$, and Dawei Zhang ${ }^{2,3 *}$ \\ ${ }^{1}$ School of Biological Engineering, Dalian Polytechnic University, Dalian 116034, P.R. China \\ ${ }^{2}$ Tianjin Institute of Industrial Biotechnology, Chinese Academy of Sciences, Tianjin 300308, P.R. China \\ ${ }^{3}$ Key Laboratory of Systems Microbial Biotechnology, Chinese Academy of Sciences, Tianjin 300308, P.R. China \\ ${ }^{4}$ Department of Animal Science, College of Animal Science and Veterinary Medicine, Tianjin Agricultural University, Tianjin 300170, P.R. China
}

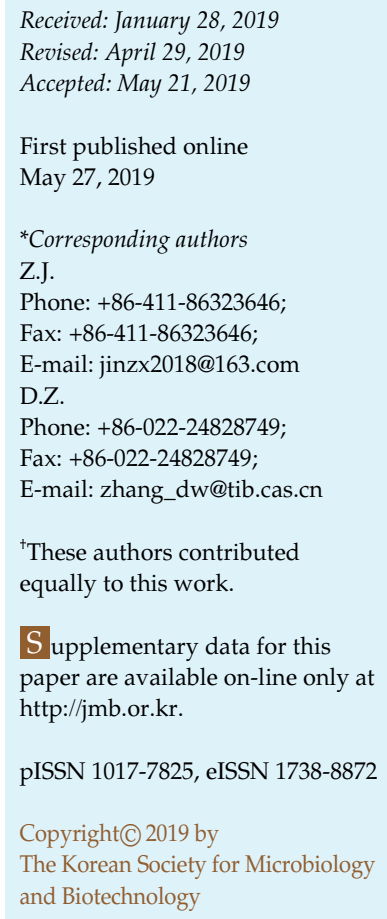

Current strategies of strain improvement processes are mainly focused on enhancing the synthetic pathways of the products. However, excessive metabolic flux often creates metabolic imbalances, which lead to growth retardation and ultimately limit the yield of the product. To solve this problem, we applied a dynamic regulation strategy to produce L-phenylalanine (LPhe) in Escherichia coli. First, we constructed a series of Phe-induced promoters that exhibited different strengths through modification of the promoter region of tyrP. Then, two engineered promoters were separately introduced into a Phe-producing strain xllp1 to dynamically control the expression level of one pathway enzyme AroK. Batch fermentation results of the strain xllp3 showed that the titer of Phe reached $61.3 \mathrm{~g} / \mathrm{l}$ at $48 \mathrm{~h}$, representing a titer of 1.36fold of the strain xllp1 (45.0 g/l). Moreover, the L-Phe yields on glucose of xllp3 $(0.22 \mathrm{~g} / \mathrm{g})$ were also greatly improved, with an increase of 1.22-fold in comparison with the xllp1 (0.18 g/ g). In summary, we successfully improved the titer of Phe by using dynamic regulation of one key enzyme and this strategy can be applied for improving the performance of strains producing other aromatic amino acids and derived compounds.

Keywords: L-Phenylalanine, E. coli, dynamic regulation, AroK, fermentation

\section{Introduction}

Considerable progress in metabolic engineering has been achieved in recent decades, allowing rational and accurate engineering in producing strains [1-3]. However, the yield and productivity of targeted products are often constrained by metabolic imbalances [4]. Extremely low pathway gene expression results in bottlenecks for product formation, whereas excessively high pathway gene expression leads to waste of cellular resources that were originally expected to channel into the target products. In addition, an overdose of the pathway enzymes or intermediate metabolites causes cell injury during fermentation [5].
Organisms have evolved to exhibit abilities to dynamically readjust the metabolic flux according to the physiological state in real time. Inspired by this, a well-engineered strain can sense critical cytoplasmic pathway intermediates and deliver the intermediates at optimal levels to maximize the productivity and yield when the environment of the strain changes [6, 7]. However, as a trade-off occurs between the production of target products and the accumulation of biomass in the industrial strain [8], one major challenge in attaining such an ideal state is to achieve a perfect balance between cell growth and product synthesis [9-13]. For instance, Ding et al. [14] selected one mutant through multiple random mutagenesis of E. coli W3110 and over- 
expressed $\operatorname{aroF}$, phe $A^{\mathrm{fbr}}$, and aroA, and the L-Phe titer of the engineered strain reached $62.47 \mathrm{~g} / 1$ in a 5 - $\mathrm{L}$ fed-batch fermentation, with the conversion rate of $25.74 \% \mathrm{~mol} / \mathrm{mol}$ glucose. Liu et al. [15] obtained a W3110-derived L-tyrosine auxotrophic strain through mutation breeding and overexpressed aroF, aroD, and $p h e A^{\mathrm{fbr}}$. Then, the TyrR was inactivated and galP and glk were integrated in the chromosome of the engineered strain. Via system-level engineering, the L-Phe titer of the final strain reached $72.9 \mathrm{~g} / \mathrm{l}$ in a 5-L fermenter under non-optimized fermentation conditions.

L-Phenylalanine (L-Phe), an essential amino acid for humans and other animals, has been widely used in the food, feed additive and pharmaceutical industries. In particular, Phe is used as the precursor for the production of aspartame in the food industry [16]. Over the past years, microbial production of Phe has attracted much attention. Therefore it is significant to construct an industrially excellent strain for efficient production of Phe [8, 14, 15]. The biosynthetic pathways for the production of aromatic amino acid in Escherichia coli are shown in Fig. 1. Successful metabolic engineering work for overproducing Phe in E. coli is mainly focused on increasing the supplement of Phe precursors (such as phosphoenolpyruvate (PEP) and erythrose-4-phosphate (E4P)), preventing the carbon flow towards branch pathways and enhancing the Phe export [14, 17-19]. In addition, further enhancing the metabolic flux towards Phe is attained by removing the transcriptional control points and relieving rate-limiting steps in the shikimate (SHK) pathway. The conversion from SHK to SHK-3-phosphate (S3P) catalyzed by SHK kinase isoenzymes I and II (encoded by aroK and aroL, respectively) is also regarded as a rate-limiting step for Phe synthesis [1, $17,20,21]$. In our previous study, we obtained a Phehyperproducing strain (named xllp1) that was derived from E. coli W3110 strain and then modified through stepwise rational metabolic engineering. When aroK was overexpressed in xllp1, the Phe titer of the recombinant strain was slightly increased, but the strain's growth was significantly affected. We assumed that AroK is a potential bottleneck in this strain, but it would damage cell growth by simply overexpressing AroK with a constitutive promoter. Therefore, the expression level of AroK needed to be dynamically controlled in xllp1.

To establish a dynamic system for regulating the transcription of aroK in real time in a Phe-producing strain, the first step was to develop a Phe-biosensor that responds to the variation in intracellular Phe levels. Thus, we attempted to apply the naturally occurring Phe-sensing protein TyrP. Through modulating the promoter regions, we constructed a series of $\operatorname{tyr} P$ promoter mutants that exhibited different strengths and activation effects by Phe. Next, we verified the feasibility of this strategy by controlling the expression level of AroK in xllp1. We substituted the natural promoter of AroK $\left(\right.$ AroK $\left._{\mathrm{WT}}\right)$ with the engineered promoters in xllp1 and analyzed the titer and yield of Phe after fermentation.

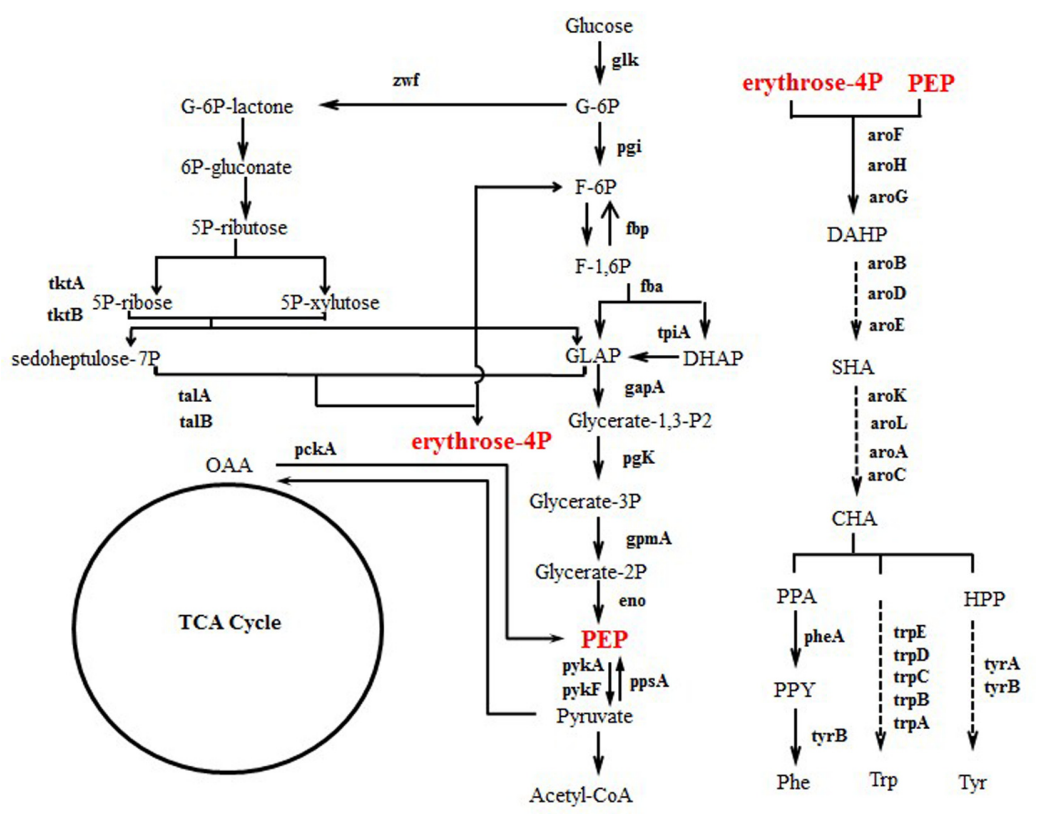

Fig. 1. Biosynthetic pathways for the production of L-Phe and other aromatic amino acids in Escherichia coli. 
Table 1. Strains and plasmids.

\begin{tabular}{|c|c|c|}
\hline Strains or plasmids & Relevant characteristics & Sources of reference \\
\hline \multicolumn{3}{|l|}{ Strains } \\
\hline E. coli DH5 $\alpha$ & This strain was used for the cloning of the plasmids. & Our laboratory \\
\hline xllp1 & $\begin{array}{l}\text { Phe-overproducing strain. This strain was derived from E. coli W3110 strain, which was initially } \\
\text { subject to multiple random mutagenesis. }\end{array}$ & Our laboratory [14] \\
\hline xllp2 & xllp1 derivative, obtained by replacing the aroK's natural promoter with promoter P8 & This study \\
\hline xllp3 & xllp1 derivative, obtained by replacing the aroK's natural promoter with promoter P9 & This study \\
\hline xllp4 & $\begin{array}{l}\text { xllp1 derivative, obtained by replacing the aroK's natural promoter with an artificial promoter } \\
\text { from the MIT parts registry }\end{array}$ & This study \\
\hline \multicolumn{3}{|l|}{ Plasmids } \\
\hline pUC18-P1 & Derivative from $\mathrm{pUC} 18$, containing promoter $\mathrm{P} 1$ and $y f p$ as a reporter gene. & This study \\
\hline pUC18-P2 & Derivative from $\mathrm{pUC} 18$, containing promoter $\mathrm{P} 2$ and $y f p$ as a reporter gene. & This study \\
\hline pUC18-P3 & Derivative from $\mathrm{pUC} 18$, containing promoter $\mathrm{P} 3$ and $y f p$ as a reporter gene. & This study \\
\hline pUC18-P4 & Derivative from $\mathrm{pUC} 18$, containing promoter $\mathrm{P} 4$ and $y f p$ as a reporter gene. & This study \\
\hline pUC18-P5 & Derivative from $\mathrm{pUC} 18$, containing promoter $\mathrm{P} 5$ and $y f p$ as a reporter gene. & This study \\
\hline pUC18-P6 & Derivative from $\mathrm{pUC} 18$, containing promoter $\mathrm{P} 6$ and $y f p$ as a reporter gene. & This study \\
\hline pUC18-P7 & Derivative from $\mathrm{pUC18}$, containing promoter $\mathrm{P} 7$ and $y f p$ as a reporter gene. & This study \\
\hline pUC18-P8 & Derivative from $\mathrm{pUC} 18$, containing promoter $\mathrm{P} 8$ and $y f p$ as a reporter gene. & This study \\
\hline pUC18-P9 & Derivative from $\mathrm{pUC} 18$, containing promoter $\mathrm{P} 9$ and $y f p$ as a reporter gene. & This study \\
\hline pUC18-P10 & Derivative from pUC18, containing promoter P10 and $y f p$ as a reporter gene. & This study \\
\hline pUC18-P11 & Derivative from pUC18, containing promoter P11 and $y f p$ as a reporter gene. & This study \\
\hline pUC18-P12 & Derivative from pUC18, containing promoter P12 and $y f p$ as a reporter gene. & This study \\
\hline
\end{tabular}

We expected that the strain would grow well in the early growth stage and the Phe synthetic pathway could be activated by the increased concentrations of intracellular Phe in the middle and late phases of fermentation.

\section{Material and Methods}

\section{Bacterial Strains Plasmids and Cultivation Conditions}

Strains and plasmids used in this study are listed in Table 1. E. coli $\mathrm{DH} 5 \alpha$ strains were purchased from Transgene (China) and used for cloning. xllp1 was derived from E. coli W3110 and stored in our lab [14]. The genome information of xllp2, xllp3, and xllp4 is also listed in Table 1. The CRISPR/Cas9 genomic editing system was a kind gift from Dongdong Zhao [22]. All primers were synthesized in Genewiz (China) and the primer sequences are listed in Table S1.

For shake flask fermentation, a single colony was selected for inoculation in $5 \mathrm{ml}$ of LB medium overnight at $37^{\circ} \mathrm{C}$. The strains were then inoculated into a $250-\mathrm{ml}$ shake flask containing $20 \mathrm{ml}$ of seed culture medium $\left(4 \mathrm{~g} / 1 \mathrm{KH}_{2} \mathrm{PO}_{4}, 20 \mathrm{~g} / 1\right.$ glucose, $14 \mathrm{~g} / 1$ (NH4) ${ }_{2} \mathrm{SO}_{4}, 2 \mathrm{~g} / 1 \mathrm{MgSO}_{4}, 8 \mathrm{~g} / 1$ yeast extract, $2 \mathrm{~g} / 1$ sodium citrate, $8 \mathrm{mg} / 1 \mathrm{FeSO}_{4} \cdot 7 \mathrm{H}_{2} \mathrm{O}$ and $40 \mathrm{mg} / \mathrm{l}$ thiamine) at a ratio of 1:100 and incubated at $37^{\circ} \mathrm{C}$ for $8 \mathrm{~h}$. The culture was then transferred into the fermentation medium $\left(10 \mathrm{~g} / 1\left(\mathrm{NH}_{4}\right)_{2} \mathrm{SO}_{4}, 5 \mathrm{~g} / 1 \mathrm{KH}_{2} \mathrm{PO}_{4}, 4 \mathrm{~g} / 1\right.$ yeast extract, $20 \mathrm{~g} / \mathrm{l}$ glucose, $5 \mathrm{~g} / 1 \mathrm{MgSO}_{4}, 0.015 \mathrm{~g} / 1 \mathrm{FeSO}_{4} \cdot 7 \mathrm{H}_{2} \mathrm{O}$, $0.015 \mathrm{~g} / 1 \mathrm{MnSO}_{4} \cdot \mathrm{H}_{2} \mathrm{O}, 3 \mathrm{~g} / \mathrm{l}$ betaine, $10 \mu \mathrm{g} / \mathrm{l}$ biotin, and $250 \mathrm{~m} \mathrm{~g} / \mathrm{l}$ Tyr) at a ratio of 1:30. For each strain the process was performed in triplicate.

\section{Determination of the Intracellular Phe Concentration}

Five hundred microliters of cell suspension was collected by centrifugation in a tube containing $100 \mu \mathrm{l}$ of silicone oil. The cell pellets were then disrupted by sonication and resuspended in 200 $\mu \mathrm{l}$ of perchloric acid $(20 \%, \mathrm{v} / \mathrm{v})$. The extraction was neutralized by adding $200 \mu \mathrm{l}$ of $\mathrm{Na}_{2} \mathrm{CO}_{3}$ and then the supernatant was filtered through a membrane $(0.22 \mu \mathrm{m})$ and stored at $-20^{\circ} \mathrm{C}$ before use. The Phe concentration was determined by high-performance liquid chromatography (HPLC) analysis.

\section{CRISPR/Cas9-Mediated Genome Editing}

The genome editing procedure was conducted according to Zhao's assay [22]. Specifically, to construct pCas-aroK, the aroKspecific N20 sequences were introduced into the pRed_Cas9_ recA_ $\Delta$ poxb300 plasmid by using the primer cas-aroK-N20-LF and cas-aroK-N20-LR (Table S1) to produce pCas-aroK-N20. Homologous arms of aroK were obtained from genomic DNA of E. coli MG1655 by using the primers cas-aroK-up-LF +cas-aroK- 
up-LR and cas-aroK-down-LF +cas-aroK-down-LR (Table S1), respectively. Then, $\mathrm{pCas}-\mathrm{aroK}$ was obtained by inserting the homologous arms into the pCas-aroK-N20 by Gibson assembly assay. Subsequently, the pCas-aroK plasmid was transformed into electrocompetent cells and then cultivated overnight on lysogeny broth (LB) plates containing $0.2 \%$ arabinose. To improve the efficiency of genome editing, colonies that survived on the agar plates were recultivated in LB plates containing $0.2 \%$ arabinose. The surviving colonies were then verified by PCR analysis and DNA sequence.

\section{Real-Time PCR Analysis}

Cells that were inoculated in LB shake flask after $37 \mathrm{~h}$ were collected by centrifugation at $13,000 \mathrm{~g}$ and resuspended in the lysis buffer (China). RNA extraction and cDNA synthesis were carried out according to the manufacturer's instructions (TAKARA, Japan). Real-time PCR was performed with the ABI Prism7000 Sequence Detection System using the SYBR Green PCR Master Mix. The amplification conditions were as follows: $10 \mathrm{~min}$ at $95^{\circ} \mathrm{C}$, and a two-step cycle at $95^{\circ} \mathrm{C}$ for $15 \mathrm{sec}$ and $60^{\circ} \mathrm{C}$ for $60 \mathrm{sec}$ for a total of 45 cycles. All samples were performed in triplicate. The data were normalized using the ihfB gene as an internal control (housekeeping gene). All of the primers used in this study are shown in Table S1.

\section{Fermentation of Phe-Producing Strains in a 7-L Fermenter}

A single colony was cultivated in LB medium overnight and inoculated into the seed culture $(8 \mathrm{~g} / 1$ yeast extract, $14 \mathrm{~g} / \mathrm{l}$ $\left(\mathrm{NH}_{4}\right)_{2} \mathrm{SO}_{4}, 2 \mathrm{~g} / 1$ sodium citrate, $4 \mathrm{~g} / 1 \mathrm{KH}_{2} \mathrm{PO}_{4}, 20 \mathrm{~g} / 1$ glucose, $8 \mathrm{mg} / 1 \mathrm{FeSO}_{4} \cdot 7 \mathrm{H}_{2} \mathrm{O}, 40 \mathrm{mg} / \mathrm{VB}_{1}$, and $7 \mathrm{~g} / 1 \mathrm{MgSO}_{4}$ ) for $7 \mathrm{~h}$ at $37^{\circ} \mathrm{C}$. Then the cells were inoculated into a 7-L fermenter containing $3.5 \mathrm{~L}$ of fermentation culture $\left(20 \mathrm{~g} / 1\right.$ glucose, $10 \mathrm{~g} / 1\left(\mathrm{NH}_{4}\right)_{2} \mathrm{SO}_{4}$, $5 \mathrm{~g} / 1 \mathrm{KH}_{2} \mathrm{PO}_{4}, 5 \mathrm{~g} / 1 \mathrm{MgSO}_{4}, 4 \mathrm{~g} /$ lyeast extract, $0.015 \mathrm{~g} / 1 \mathrm{FeSO}_{4} \cdot 7 \mathrm{H}_{2} \mathrm{O}$, $0.015 \mathrm{~g} / 1 \mathrm{MnSO}_{4} \cdot \mathrm{H}_{2} \mathrm{O}$, and $\left.250 \mathrm{mg} / 1 \mathrm{Tyr}, 1: 10 \mathrm{v} / \mathrm{v}\right)$. During batchphase fermentation, the $\mathrm{pH}$ was maintained at 7.0 with $10 \%$ $\mathrm{NH}_{4} \mathrm{OH}$ solution. The temperature was initially set at $33^{\circ} \mathrm{C}$ and then increased to $38^{\circ} \mathrm{C}$ when the $\mathrm{OD}_{600}$ reached 30 . The dissolved oxygen was constantly kept at $20 \%$ saturation by cascading the agitation speed and aeration rate. After initial glucose was exhausted, the residual glucose was maintained at $1 \sim 5 \mathrm{~g} / 1$ by supplementing $600 \mathrm{~g} / 1$ glucose in the fermentation process. Samples were collected every $2 \mathrm{~h}$ to determine the $\mathrm{OD}_{600}$, the residual glucose and the concentration of Phe.

\section{HPLC Analysis of Phe Amount in the Fermentation Broth}

Phe titers were measured using HPLC with UV detection (Agilent 1260 Series). Samples collected from the shake flasks were centrifuged and the supernatant was then diluted with $\mathrm{ddH}_{2} \mathrm{O}$. HPLC analysis of Phe was carried out by using Zorbax Eclipse-AAA columns. Derivatization was performed according to the manufacturer's protocol. The mobile phase consists of phase A (40 mM Na $\left.2 \mathrm{HPO}_{4} \mathrm{pH} 7.8\right)$ and phase B (ACN: $\mathrm{MeOH}$ : $\left.\mathrm{H}_{2} \mathrm{O}=45: 45: 10\right)$. The column temperature was set at $40^{\circ} \mathrm{C}$ and the flow rate was $2 \mathrm{ml} / \mathrm{min}$. All of the experiments were carried out in triplicate.

\section{Results and Discussion}

\section{Construction of the tyrP Promoter Mutants}

The expression of TyrP is activated by TyrR in the presence of Phe. This activation requires that the TyrR binds to the two adjacent TyrR boxes separated by one base in the tyrP promoter [23]. In addition, the weak box overlaps with the RNA polymerase (RNAP) binding site in the tyrP promoter [24]. The activation function requires binding of the TyrR dimer to the strong box while the repression function requires binding of the TyrR dimer to both boxes. Because of the overlap region of the TyrR and RNAP binding sites in the promoter of tyrP, we assumed that by altering the bases in the promoter region may disturb the interaction between RNAP, TyrR and tyrP promoter. The three-base insertion between the two TyrR boxes has been reported to eliminate repression and enhance the level of activation by TyrR [25]. In addition, we introduced individual or combined conversed bases into the -35 region $(-38 \mathrm{~T}-\mathrm{A},-37 \mathrm{~T}-\mathrm{A})$ or -10 region $(-15 \mathrm{~T}-\mathrm{G}$, $-13 \mathrm{~A}-\mathrm{T},-12 \mathrm{C}-\mathrm{A},-11 \mathrm{C}-\mathrm{A})$ to alter the dynamic response of the promoter towards Phe [24]. Finally, Andrews et al. showed that because of the higher melting of the tyrP promoter on supercoiled DNA, it can form a more stable complex with RNAP than the linear promoter, therefore we attempted to insert three $\mathrm{A} / \mathrm{T}$ bases into the discriminator box to enhance the stability of the complex [25]. According to the above content, we altered the bases in -35 region, -10 region, the discriminator and the number of bases between the two boxes to create promoters that exhibited the different strengths and dynamic behaviors of Phe. Details on base changes in the promoter mutants are presented in Table S2. The schematic diagram of locations of SNP in the different promoter mutants is shown in Fig. 2A. All promoter mutants were separately ligated into the pUC18 harboring $y f p$ as a reporter gene. The plasmids were transformed into the E. coli DH5 $\alpha$ strain, and YFP fluorescence was detected in the logarithmic phase. Considering the strict regulation of amino acid transport systems in E. coli, we applied the dipeptide feeding assay to artificially alter the intracellular Phe level. When added into the medium, the Phe-Phe dipeptides undergo active uptake by the peptide transport systems and are then hydrolyzed by hydrolases, which can sharply improve the intracellular Phe level.

As shown in Fig. 2B, the strengths of the constructed 

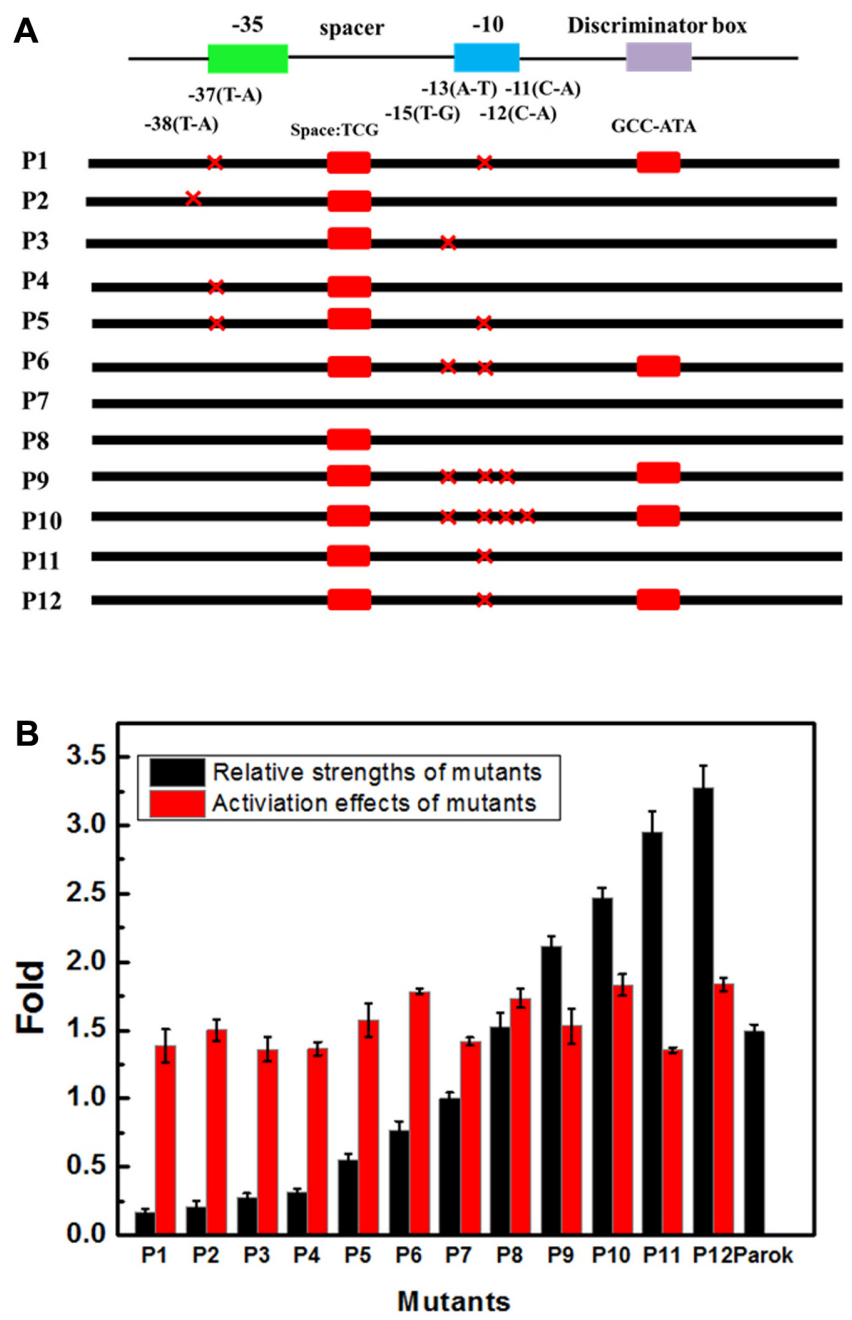

Fig. 2. Effects of mutations in the tyrP promoter regions on the promoter strengths and activation effects by Phe.

(A) The location of SNP in promoter mutants. The red " $x$ " represents base replacement and the red square represents base insertion in the designated sites. (B) Relative strengths and activation effects of promoter mutants. Error bars represent the means of the standard deviation of three replicates.

promoters are distributed evenly, ranging from 0.16-3.28 times of $\operatorname{aroK}_{W T}$. Among these promoters, six exhibited increases whereas five showed decreases in strength compared with that of the natural promoter of tyrP $\left(\operatorname{tyr} P_{W T}\right)$. In addition, we measured the activation effects of promoter mutants in response to the addition of Phe by dipeptide feeding assay. As seen in Fig. 2B, all of the mutants were activated by the addition of Phe but the activation effects of most mutants were impaired to varying degrees compared with that of $\operatorname{tyr} P_{W T}$. Mutant8, which carried a three-base insertion between the two TyrR boxes, showed a higher strength (1.53-fold) and a comparable activation effect compared with $\mathrm{TyrP}_{\mathrm{WT}}$. One possible reason is that RNAP binds more closely to mutant 8 because of its conformational changes. Meanwhile the interaction among the RNAP, TyrR protein and the promoter region is not affected by the three-base insertion. The promoter activity of mutant11, which contained one base substitution in the -10 region of mutant9, was 2.95-fold higher compared with $\operatorname{TyrP}_{\mathrm{wT}}$, but the activation effect by TyrR was significantly dropped. Besides, altering the base in -35 region (mutant1, mutant2 and mutant4) resulted in both a significant reduction of transcription and a drastic loss of activation by TyrR. Therefore, it was concluded the -35 region played a crucial role in the TyrR-dependent activation effect in the presence of Phe. Even altering one base in this region may cause a drastic loss of activation. Interestingly, when inserting three-bases into the discriminator box in mutant11 (producing mutant12), a 3.28-fold increase in transcription was found. In contrast to mutants that contained SNP in -35 region or -10 region, mutant12 presented a strong activation by TyrR in the presence of Phe. This indicated that the three-base insertions in the discriminator box greatly promoted the TyrRdependent activation effect. However, when base changes were coexisted in the discriminator box and in -35/-10 region (mutant1, mutant6, mutant9, and mutant10), the situation became complex and the activation effects of these mutants were hard to conclude. Compared with mutant12, it was found that mutant6 presented a similar strong activation effect by TyrR but the promoter strength dropped to $76.3 \%$ of the wild type. As mentioned above, the three-base insertions between the two TyrR boxes and the three-base insertions in the discriminator box cooperatively contributed to the activation effect of this promoter by TyrR. However, introducing two base mutations in -10 region simultaneously (mutant6: $-13 \mathrm{~A}-\mathrm{T}$ and $-15 \mathrm{~T}-\mathrm{G}$ ) may damage the interaction between the RNA polymerase and the promoter sequence. This conclusion still needed follow-up study and further confirmation by relevant studies.

Subsequently, we determined that the promoter mutants were activated by Phe in a dose-dependent way. The promoter strengths of P8 and P9 were 1.12-fold and 1.42fold of the $\mathrm{P}_{\text {aroK, }}$ respectively, and the activation effects of these two mutants were not significantly impaired because of the site mutations. Therefore, they were chosen as potential candidates for substituting the natural promoter of aroK for improving Phe production in xllp1. E. coli DH5 $\alpha$ strains carrying two promoter mutants were cultivated in 


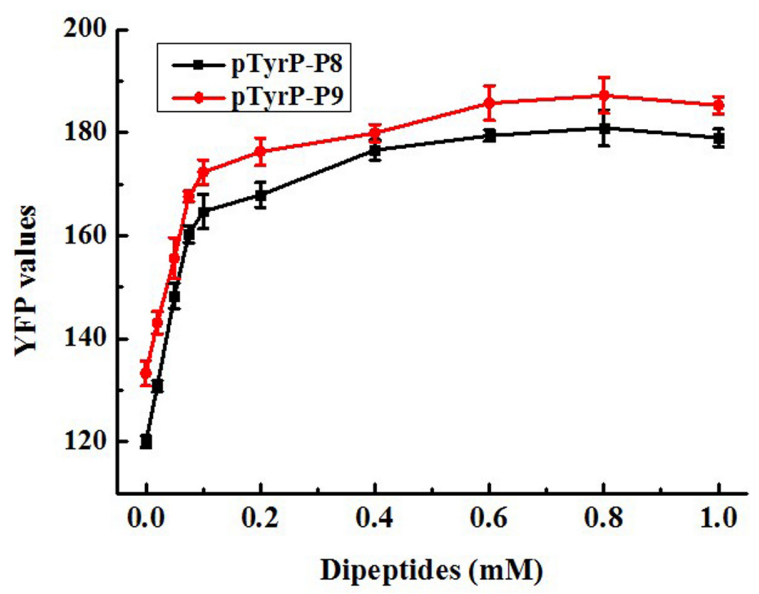

Fig. 3. Characterization of pTyrP-P8 and pTyrP-P9 in response to increasing Phe-Phe concentrations in the medium.

E. coli $\mathrm{DH} 5 \alpha$ strains carrying pTyrP-P8 and pTyrP-P9 were cultivated in $\mathrm{M} 9$ containing dipeptides ranging from $0 \mathrm{mM}$ to $1.0 \mathrm{mM}$ for $0.5 \mathrm{~h}$. Fluorescence values were detected by FCM. Error bars represent the means of the standard deviation of three replicates.

the M9 medium containing Phe-Phe dipeptides ranging from $0 \mathrm{mM}$ to $1.0 \mathrm{mM}$. Fluorescence values were detected by flow cytometry (FCM). For pTyp-p8, a clear increase in YFP value was observed when $0.05 \mathrm{mM}$ Phe-Phe was fed in the medium (Fig. 3). The YFP value grew rapidly before reaching $0.1 \mathrm{mM}$ Phe-Phe and continued to improve until $0.8 \mathrm{mM}$ Phe-Phe and remained stable thereafter. The similar phenomenon was also observed in the strains carrying pTyp-p9 plasmid in response to the Phe-Phe addition. Both curves indicated that the activation effects of the two promoter mutants remained existent and the activation effects were increased in response to the improved concentrations of Phe.

\section{Replacing the Natural Promoter of aroK by Promoter Mutants in xllp1}

The reactions catalyzed by SHK kinase are often considered as a rate-limiting step to produce Phe in E. coli $[21,26,27]$. There are two types of shikimate kinase that are separately encoded by aroL and aroK. Although AroL was regarded as the preferred shikimate kinase due to the lower $\mathrm{K}_{\mathrm{m}}$, the overexpression of aroK produced higher Phe than aroL because the affinity of AroK for shikimate is much lower than that of AroL [28, 29]. Therefore, aroK was chosen as the potential target to determine the availability of dynamic regulation on Phe production in xllp1.

The natural aroK promoter in xllp1 was replaced with the artificial promoters P8 and P9 on the basis of the CRISPER-
Cas9-mediated genome editing assay to produce strains xllp2 and xllp3, respectively. To confirm that the production of Phe was improved because of the dynamic regulation created by the promoter mutants instead of simply a change in promoter strength, a strong artificial promoter was selected from the MIT parts registry (http:// parts.igem.org/) to replace the aroK promoter in xllp1. All strains were cultivated in shake flasks for $48 \mathrm{~h}$. The OD, Phe titers in fermentation broth and intracellular Phe concentrations were measured every $8 \mathrm{~h}$. At the initial stage of fermentation, cells proliferated quickly and few products were accumulated. When the strains were at stationary phase, they stopped growing and started to produce Phe quickly. Fig. 4A showed that the growth of xllp2 and xllp3 presented no significant difference compared with that for xllp1, whereas the final OD of xllp4 was reduced to $71.8 \%$ of that of xllp1, so we therefore inferred the growth condition of xllp4 was impaired due to the high expression levels of AroK. As for the Phe production, the titers of Phe produced by xllp2 and xllp3 showed no obvious differences at the initial $24 \mathrm{~h}$ compared with that of xllp1, after that these two strains gradually accumulated higher concentrations of Phe. Compared with the xllp1 stain, the final concentrations of xllp2 and xllp3 were increased by 1.25- and 1.28-fold, reaching $5.67 \mathrm{~g} / 1$ and $5.82 \mathrm{~g} / 1$ respectively. However, the Phe production rate of xllp4 strain was kept at a relatively low level through the fermentation process, especially in the late fermentation stage. The production of xllp4 strain was only $3.48 \mathrm{~g} / 1$ at the end of fermentation (Fig. 4B). In addition, the Phe yield of xllp2 and xllp3 reached 0.222 and $0.231 \mathrm{~g} \mathrm{~g}^{-1}$ glucose, a significant increase compared with xllp1 and xllp4, which were with 0.183 and $0.179 \mathrm{~g} \mathrm{~g}^{-1}$ glucose, respectively. The intracellular Phe concentrations of the four strains were almost zero at the early stage of fermentation and increased sharply after $24 \mathrm{~h}$ of fermentation (Fig. 4C). After that, xllp2 and xllp3 continued to accumulate higher intracellular concentrations of Phe while xllp1 and xllp4 almost kept stable until the end of fermentation. The final Phe amount in xllp1 and xllp4 reached to 47.1 and $52.6 \mathrm{mg} / \mathrm{l}$ in $32 \mathrm{~h}$, respectively. By contrast, the final intracellular Phe in xllp2 and xllp3 reached 103.6 and $118.2 \mathrm{mg} / \mathrm{l}$, respectively. The Pheinduced promoters in this study were designed to respond to the intracellular Phe instead of the Phe accumulated in the medium, so the higher concentrations of intracellular Phe in xllp2 and xllp3 provide direct evidence that the Pheinduced promoters work as intended in vivo. Of course, the higher extracellular concentrations of Phe of xllp2 and xllp3 also suggest that we successfully improved the titer 

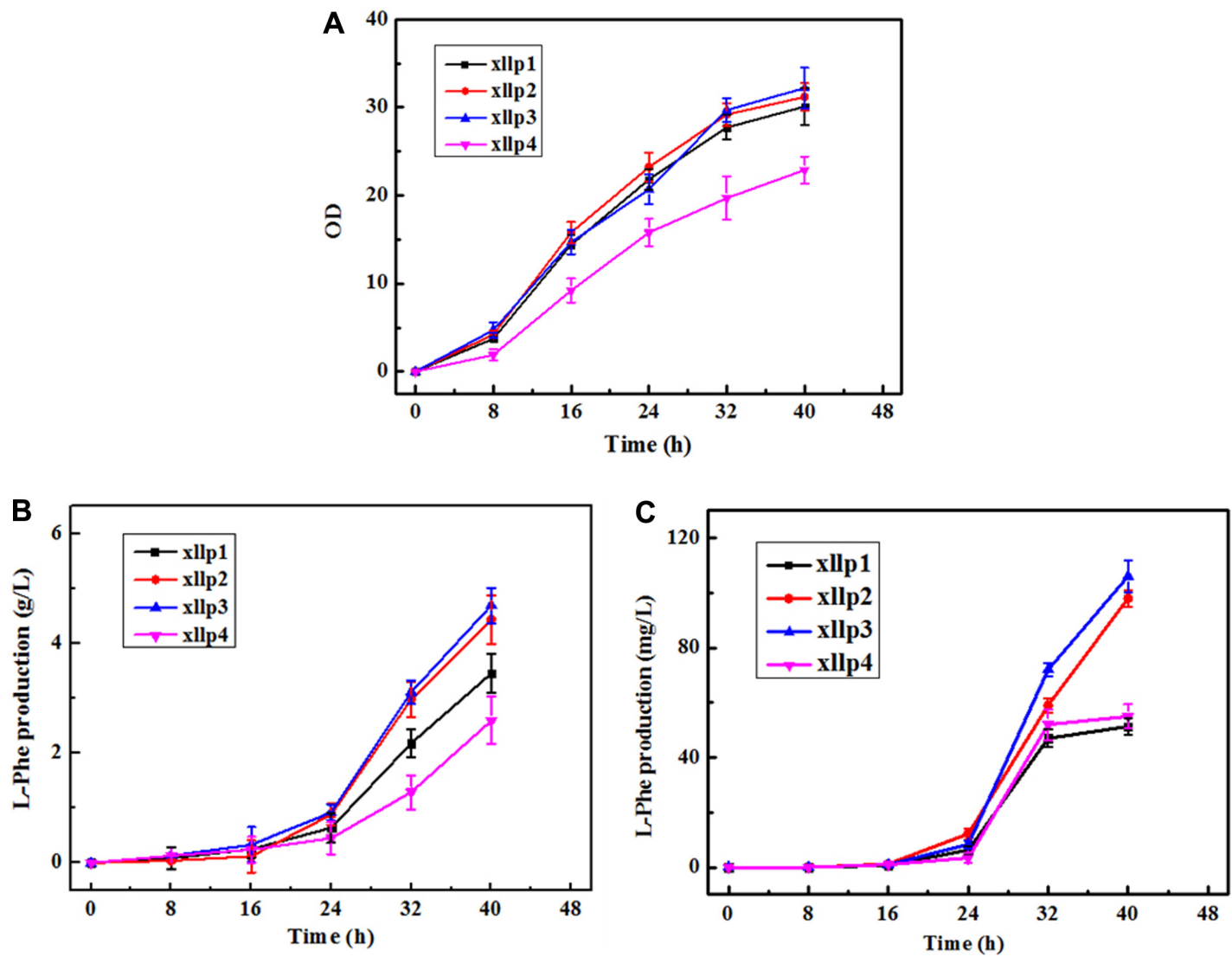

Fig. 4. Fermentation profiles of xllp1, xllp2, xllp3, and xllp4.

Samples were collected every $8 \mathrm{~h}$ during the fermentation process. The OD of each of the four strains was detected by spectrophotometry (a). The extracellular (B) and intracellular (C) concentrations of Phe produced by the recombinant strains were measured by HPLC.

of Phe by using dynamic regulation of AroK. So, both the intracellular and the extracellular concentrations of Phe were necessary for a clear explanation of the function of dynamic regulation in xllp2 and xllp3.

\section{Quantitative Real-Time PCR Analysis of Genes Involved in Phe Synthesis Pathway in xllp2 and xllp3}

To further verify the functionality of the promoter mutants on the improvement of Phe in xllp2 and xllp3, we investigated the transcription levels of the pathway enzymes involved in Phe biosynthesis in E. coli. Fig. 4C showed that the concentrations of intracellular Phe were much higher in xllp2 and xllp3 than that of xllp1 in $37 \mathrm{~h}$, so samples in this time were collected to investigate the transcriptional changes of aroK, aro $A$, aro $C$, phe $A$, and tyr $B$ by real-time PCR. As expected, the transcription levels of aroK in xllp2 and xllp3 were separately increased by 3.2fold and 3.8-fold compared with xllp1 (Fig. 5). The other genes, including aro $A$, aro $C$, phe $A$, and $\operatorname{tyr} B$, were also upregulated at different degrees in xllp2 and xllp3 than in xllp1. Therefore, the aroK gene in xllp2 and xllp3 was clearly transcriptionally activated by the high intracellular Phe level, rendering higher carbon flux toward the Phe synthetic pathway. This also led to the transcription upregulation of other genes in this pathway. According to the fermentation performance of xllp2 and xllp3 in the shake flask, we chose xllp3 as the best industrial strain for subsequent evaluation in the 7-L fermenter.

\section{Fermentation of xllp3 in a 7-L Fermenter}

We performed a fed-batch fermentation with intermittent glucose feeding to measure the production of xllp1 and xllp3 strains in a 7-L fermenter. As shown in Fig. 6, the growth trends of xllp1 and xllp3 were similar except that xllp3 exhibited a slight decrease in $\mathrm{OD}_{600}$ from $38 \mathrm{~h}$ to $42 \mathrm{~h}$ after fermentation. The $\mathrm{OD}_{600}$ of xllp1 and xllp3 was roughly equal at the end of fermentation. As for the Phe production, the Phe yield of xllp1 and xllp3 showed a 


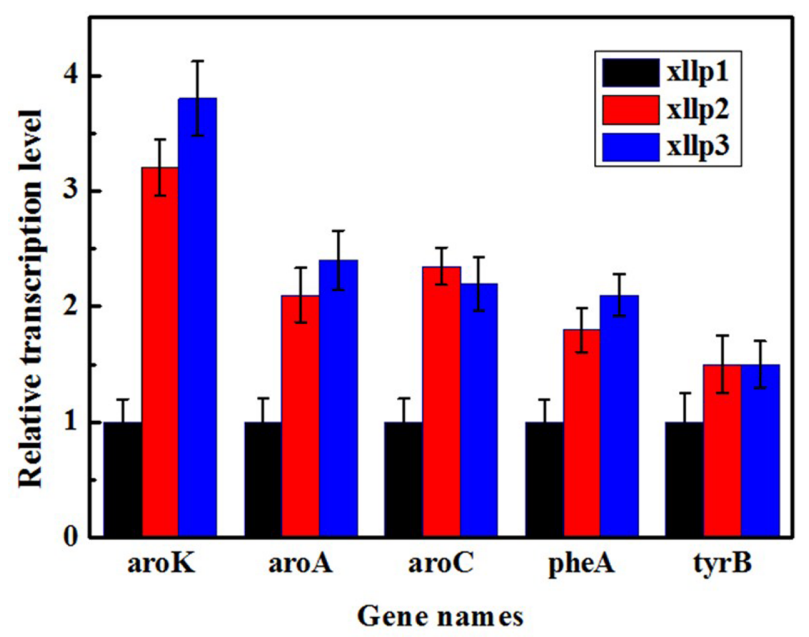

Fig. 5. Relative transcription levels of genes involved in the Phe synthetic pathway in xllp1, xllp2, and xllp3, as determined by real-time PCR.

Experiments were conducted in triplicate. Error bars represent the means of the standard deviation of three replicates.

similar trend before $32 \mathrm{~h}$ of fermentation. However, the Phe titer of xllp3 showed a rapid increase until the end of fermentation while Phe production of xllp1 was almost stable after $32 \mathrm{~h}$. The final production of xllp3 was $61.3 \mathrm{~g} / 1$ at $48 \mathrm{~h}$, with a yield of $0.22 \mathrm{~g} / \mathrm{g}$ glucose. By contrast, xllp1 achieved a titer of $45.0 \mathrm{~g} / 1$ Phe, with a yield of $0.186 \mathrm{~g} / \mathrm{g}$ glucose. This demonstrated that the dynamic overexpression of the aroK in xllp3 had a sustained activation effect for Phe overproducing when the intracellular Phe started to accumulate after $32 \mathrm{~h}$ of fermentation. It is worth noting that the growth condition of xllp1 and xllp3 was basically consistent during the process of fermentation, indicating that the dynamic overexpression of aroK in xllp3 didn't lead to the metabolic imbalance.

According to the fermentation results of xllp1, xllp2, xllp3, and xllp4, we inferred that it failed to improve the production of target compounds by simply overexpressing the key enzymes, because such overexpression can lead to metabolic imbalances, which could impair cell growth and ultimately reduce production of target compounds. In comparison, it was reasonable to improve the the titers and yields of products with the dynamic control of the key enzymes according to the environmental change. We assumed that as more intracellular Phe was accumulated, the promoter mutant was strongly activated to enhance the expression of aroK, which in turn activated the synthetic pathway for Phe formation. Therefore, the dynamic regulation in xllp2 and xllp3 facilitated the carbon flux toward the Phe synthetic pathway in a time-dependent manner instead of simply enhancing the promoter strength of the key enzymes for Phe formation.

With the progress of synthetic biology and systems biology, the production of Phe in E. coli has seen great achievements by improving the supply of precursors, alleviating the transcriptional repression, deregulating the feedback inhibition by Phe, knocking out the branch pathways and enhancing the Phe export etc. Although the Phe production was enhanced at an overall level, less attention has been paid to the optimization of the expression levels of key enzymes, which affects the amount of final product to a large extent. Previous study often
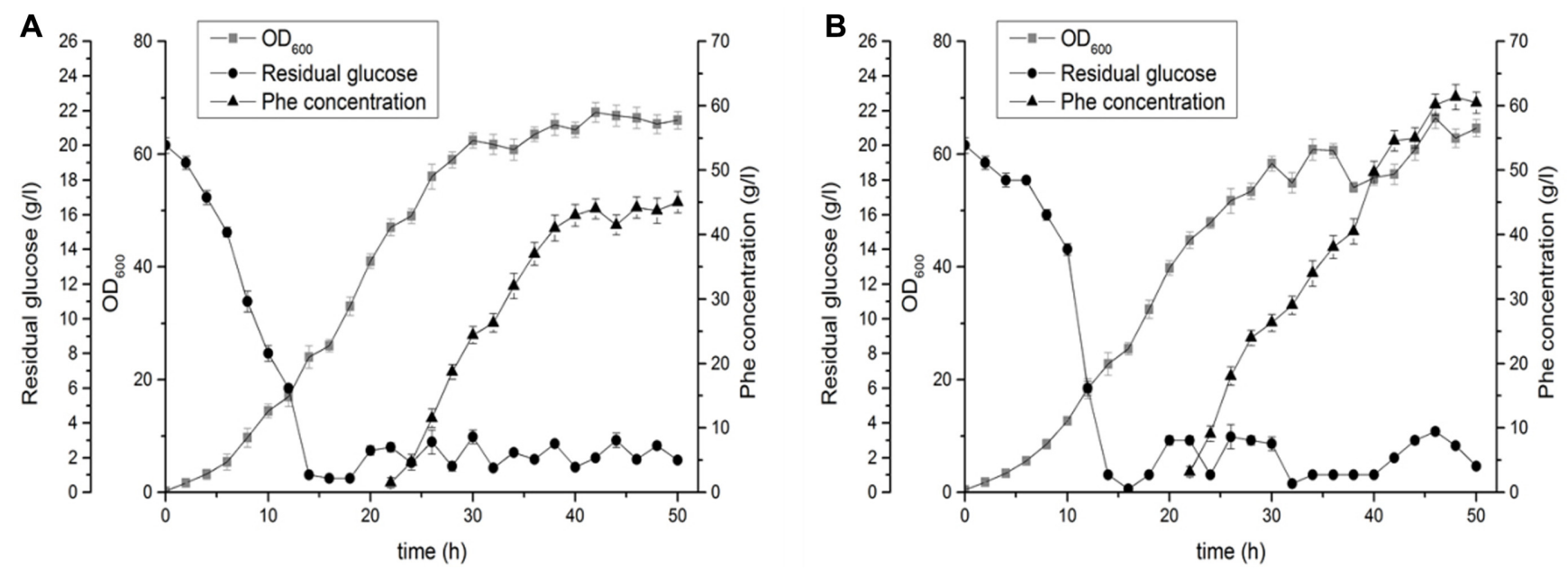

Fig. 6. Time course of Phe production by strain xllp1(a) and xllp3(b) in a 7-L fermenter.

The Phe concentration, $\mathrm{OD}_{600}$ and residual sugar were measured during cultivation. The data were obtained from three experimental replicates. 
altered the gene expression levels by introducing a serial of 5 -untranslated region (5'-UTR) for each gene of interest and determined the best candidate by analyzing the titer and yield of the product, which is obviously time-consuming and laborious $[8,30]$. Compared with this strategy, the choice of the dynamic promoters ensures that the concentration of various intermediates in the cell is in a relatively balanced state, so the risk of metabolic imbalance can be kept to a lower level. In the initial stage, dynamic promoters show little or no response to the low concentrations of intracellular product. They maintain the level of intermediate sufficient for growth and won't lead to any damage to cells. In the stationary phase of fermentation the intermediate is not needed for growth, and then the dynamic promoter's strength is strongly activated to drive more carbon flux towards product synthesis. This in turn further activates the dynamic promoters for more products. In this paper, the expression levels of key enzymes were dynamically controlled and the concentration of L-Phe was successfully increased (1.36fold) by introducing an engineered promoter into Pheproducing strain xllp1. The method used in the study is more economical and labor efficient. We believe that there is still great room to improve the yield and titer of Phe for xllp2 and xllp3. Moreover, this method has certain universality and can be applied to different strain modifications.

\section{Acknowledgments}

This work was supported by the National Key R\&D Program of China (2018YFD0901001), the Tianjin Science Fund for Distinguished Young Scholars (17JCJQJC45300), the Natural Science Foundation of China (31670604 and 31570303), the Natural Science Foundation of Liaoning Province of China (20180550237), the Natural Science Foundation of Tianjin City (CN) (16JCYBJC23500) and the Science and Technology Service Network (STS) Initiative of the Chinese Academy of Sciences (CAS), and Science and Technology Project of Tianjin (14ZCZDSY00157).

\section{Conflict of Interest}

The authors have no financial conflicts of interest to declare.

\section{References}

1. Liu SP, Zhang L, Mao J, Ding ZY, Shi GY. 2015. Metabolic engineering of Escherichia coli for the production of phenylpyruvate derivatives. Metab. Eng. 32: 55-65.
2. Xu Y, Chu H, Gao C, Tao F, Zhou Z, Li K, et al. 2014. Systematic metabolic engineering of Escherichia coli for highyield production of fuel bio-chemical 2,3-butanediol. Metab. Eng. 23: 22-33.

3. Lee SY, Kim HU. 2015. Systems strategies for developing industrial microbial strains. Nat. Biotechnol. 33: 1061-1072.

4. Keasling JD. 2010. Manufacturing molecules through metabolic engineering. Science 330: 1355-1358.

5. Dahl RH, Zhang F, Alonso-Gutierrez J, Baidoo E, Batth TS, Redding-Johanson AM, et al. 2013. Engineering dynamic pathway regulation using stress-response promoters. Nat. Biotechnol. 31: 1039-1046.

6. Sinha J, Reyes SJ, Gallivan JP. 2010. Reprogramming bacteria to seek and destroy an herbicide. Nat. Chem. Biol. 6: 464-470.

7. Fowler CC, Brown ED, Li Y. 2010. Using a riboswitch sensor to examine coenzyme $\mathrm{B}_{12}$ metabolism and transport in E. coli. Chem. Biol. 17: 756-765.

8. Liu Y, Zhuang Y, Ding D, Xu Y, Sun J, Zhang D. 2017. Biosensor-based evolution and elucidation of a biosynthetic pathway in Escherichia coli. ACS Synth. Biol. 6: 837-848.

9. Zhang F, Carothers JM, Keasling JD. 2012. Design of a dynamic sensor-regulator system for production of chemicals and fuels derived from fatty acids. Nat. Biotechnol. 30: 354-359.

10. Moser F, Espah Borujeni A, Ghodasara AN, Cameron E, Park Y, Voigt CA. 2018. Dynamic control of endogenous metabolism with combinatorial logic circuits. Mol. Syst. Biol. 14: e8605.

11. Tan SZ, Manchester S, Prather KL. 2016. Controlling central carbon metabolism for improved pathway yields in saccharomyces cerevisiae. ACS Synth. Biol. 5: 116-124.

12. Gupta A, Reizman IM, Reisch CR, Prather KL. 2017. Dynamic regulation of metabolic flux in engineered bacteria using a pathway-independent quorum-sensing circuit. Nat. Biotechnol. 35: 273-279.

13. Reizman IM, Stenger AR, Reisch CR, Gupta A, Connors NC, Prather KL. 2015. Improvement of glucaric acid production in E. coli via dynamic control of metabolic fluxes. Metab. Eng. Commun. 2: 109-116.

14. Ding D, Liu Y, Xu Y, Zheng P, Li H, Zhang D, et al. 2016. Improving the production of L-phenylalanine by identifying key enzymes through multi-enzyme reaction system in vitro. Sci. Rep. 6: 32208.

15. Liu Y, Xu Y, Ding D, Wen J, Zhu B, Zhang D. 2018. Genetic engineering of Escherichia coli to improve L-phenylalanine production. BMC Biotechnol. 18: 5 .

16. Alberto Rodriguez JAM, Noemí Flores, Adelfo Escalante, Guillermo Gosset and Francisco Bolivar. 2014. Engineering Escherichia coli to overproduce aromatic amino acids and derived compounds. Microb. Cell Fact. 13: 126.

17. Liu SP, Xiao MR, Zhang L, Xu J, Ding Z-Y, Gu Z-H, et al. 2013. Production of L-phenylalanine from glucose by metabolic engineering of wild type Escherichia coli W3110. Process Biochem. 48: 413-419. 
18. Zhou H, Liao X, Wang T, Du G, Chen J. 2010. Enhanced 1-phenylalanine biosynthesis by co-expression of $p h e A^{\mathrm{Fbr}}$ and aroF $F^{\mathrm{Wt}}$. Bioresour. Technol. 101: 4151-4156.

19. Khamduang M, Packdibamrung K, Chutmanop J, Chisti $Y$, Srinophakun P. 2009. Production of L-phenylalanine from glycerol by a recombinant Escherichia coli. J. Ind. Microbiol. Biotechnol. 36: 1267-1274.

20. Zhang Y, Meng Q, Ma H, Liu Y, Cao G, Zhang X, et al. 2015. Determination of key enzymes for threonine synthesis through in vitro metabolic pathway analysis. Microb. Cell Fact. 14: 86.

21. Krämer M, Bongaerts J, Bovenberg R, Kremer S, Müller U, Orf $\mathrm{S}$, et al. 2003. Metabolic engineering for microbial production of shikimic acid. Metab. Eng. 5: 277-283.

22. Zhao D, Yuan S, Xiong B, Sun H, Ye L, Li J, et al. 2016. Development of a fast and easy method for Escherichia coli genome editing with CRISPR/Cas9. Microb. Cell Fact. 15: 205.

23. Yang J, Ganesan S, Pittard A J. 1993. A genetic analysis of various functions of the TyrR protein of Escherichia coli. J. Bacteriol. 175: 1767-1776.

24. Yang J, Hwang JS, Camakaris H, Irawaty W, Ishihama A, Pittard J. 2004. Mode of action of the TyrR protein: repression and activation of the tyrP promoter of Escherichia coli. Mol. Microbiol. 52: 243-256.
25. Andrews AE DB, Lawley B, Cobbett C, Pittard AJ. 1991. Importance of the position of TYR R boxes for repression and activation of the tyrP and aroF genes in Escherichia coli. J. Bacteriol. 173: 5079-5085.

26. Liu SP, Liu RX, Xiao MR, Zhang L, Ding ZY, Gu ZH, et al. 2014. A systems level engineered $E$. coli capable of efficiently producing L-phenylalanine. Process Biochem. 49: 751-757.

27. Dell KA, Frost JW. 1993. Identification and removal of impediments to biocatalytic synthesis of aromatics from Dglucose: Rate-limiting enzymes in the common pathway of aromatic amino acid biosynthesis J. Am. Chem. Soc. 115: 11581-11589.

28. Oldiges M, Kunze M, Degenring D, Sprenger GA, Takors R. 2004. Stimulation, monitoring, and analysis of pathway dynamics by metabolic profiling in the aromatic amino acid pathway. Biotechnol. Prog. 20: 1623-1633.

29. Lutke-Eversloh T, Stephanopoulos G. 2008. Combinatorial pathway analysis for improved L-tyrosine production in Escherichia coli: identification of enzymatic bottlenecks by systematic gene overexpression. Metab. Eng. 10: 69-77.

30. Kim SC, Min BE, Hwang HG, Seo SW, Jung GY. 2015. Pathway optimization by re-design of untranslated regions for L-tyrosine production in Escherichia coli. Scientific Rep. 5: 13853. 